\title{
Tallas de captura de Prochilodus mariae en la parte alta del río Meta, Orinoquia colombiana
}

\author{
Capture lengths of Prochilodus mariae in the upper of the Meta \\ River, Colombian Orinoquia
}

\section{Comprimentos de captura de Prochilodus mariae na parte alta do río Meta, Orinoquia colombiana}

\author{
Hernando Ramírez-Gil ${ }^{1 *}$; Rosa E. Ajiaco-Martínez ${ }^{2 *}$; Carlos Barreto-Reyes ${ }^{3 *}$ \\ 1 BM, MSc, PhD \\ 2 BM, MSc \\ 3 BM \\ * Grupo de Investigación Evaluación, Manejo y Conservación de Recursos Hidrobiológicos y Pesqueros, Instituto de \\ Ciencias Ambientales de la Orinoquia Facultad de Ciencias Básicas, Universidad de los Llanos, Villavicencio, Colombia \\ Email: hramirezgil@gmail.com
}

Recibido: julio 21 de 2015

Aceptado: diciembre 04 de 2015

\section{Resumen}

A partir del análisis del comportamiento histórico (1980 a 2011) de las tallas de captura y tallas de madurez de hembras y machos de Prochilodus mariae, se pretendió establecer si sobre la población de la parte alta del río Meta, se presentaba algún tipo de afectación evidenciable en las tallas medias de captura anual (TMCA) y tallas de madurez (TMG). La información fue colectada mediante muestreos a los desembarcos en Puerto López, Meta. Cada ejemplar fue medido, pesado y sexado y a partir de 13444 registros, se estimaron la TMCA y las tallas de captura mensuales (TMCM); estos estimativos se compararon entre años; se hicieron análisis de correlación con los niveles del río Metica y las anomalías ENSO. Se estimó la TMG al 25\%, 50\% y $75 \%$ por año. El rango de las tallas de captura comercial de $P$. marie fue de 14 a $45 \mathrm{~cm}$ longitud estándar (LE), con diferencia entre machos y hembras. La mayor TMCA de hembras (32,6 cm LE) y machos (32 cm LE) se presentó en el año 1980, en los años siguientes este parámetro oscilo entre 30 y 26,4 cm LE en hembras y entre 26 y 29 cm LE en machos. En el ciclo anual las mayores TMCM de hembras se presentaron de abril a junio y de machos en mayo y junio. La TMGL50 de hembras, fue significativamente mayor en el año 1980 (33,5 cm LE), en los años siguientes osciló entre 27,1 y 31,8 cm LE en hembras; en machos durante todo el periodo de estudio vario entre 25,8 y 29,5 cm LE. La población de $P$. marie bajo presión de pesca en la zona de estudio, no se encuentra afectada por sobrepesca al crecimiento ni al reclutamiento.

Palabras clave: Pesquerías fluviales, tallas de madurez gonadal, especies migratorias, dinámica poblacional.

\begin{abstract}
Based on historical data of catch size and size at maturity of Prochilodus mariae obtained from 1980 to 2011, we aimed to establish if the average catch length per year and length at maturity of the population of the upper Meta River have ex-
\end{abstract}


perienced significant changes. Gathering data from fishing boats landing at Puerto Lopez, Meta, Colombia, we measured, weighed and sexed each caught specimen, obtaining 13444 records used to estimate average catches per year and per month, compared them annually, and performed correlation analyses between Metica River levels and ENSO anomalies. Length was estimated at 25\%,50\% and 75\% maturity for each year. Commercial catch length of $P$. mariae ranged from 14 to $45 \mathrm{~cm} \mathrm{SL}$, with differences in sex-specific length. The greatest average catch length for both females and males, 32,6 and $32 \mathrm{~cm}$ SL respectively, was observed in 1980, whereas in later years it varied in the range of 30 to $26,4 \mathrm{~cm}$ SL for females and 29 to $26 \mathrm{~cm}$ SL for males. The annual catch length cycle showed that the greatest average lengths occurred from April to June for females and May to June for males. Length at first maturity (L50) of females peaked at 33,5 cm SL in 1980, a significantly greater size than that observed in later years when it fluctuated between 27,1 and $31,8 \mathrm{~cm} \mathrm{SL}$. L50 of males remained within 25,8 and 29,5 cm SL throughout the period of study. Our findings show no overfishing impact on the growth and recruitment of $P$. mariae populations under fishing pressure within the area of study.

Key words: River fisheries, length of gonadal maturity, migratory species, populations dynamics.

\section{Resumo}

A partir da análise do comportamento histórico (1980-2011) dos comprimentos de captura e tamanhos de maturidade das fêmeas e machos de Prochilodus mariae, pretendeu-se estabelecer se sobre a população do alto rio Meta, apresentava-se algum tipo de afetação evidenciável no comprimento médio das capturas anuais (TMCA) e tamanho de maturação (TMG). A informação foi coletada por meio de amostragem dos desembarques em Puerto Lopez, Meta. Cada exemplar foi medido, pesado e sexado, e a partir de 13444 registros, foi estimada a TMCA e tamanho da captura mensal (TMCM); estas estimativas foram comparadas entre os anos; análise de correlação com o nível do rio Metica e anomalias ENSO foram feitas. Foi estimada o comprimento de madures ao $25 \%$, 50\% e $75 \%$ por ano. O tamanho de captura comercial de $P$. marie variou entre 14 a $45 \mathrm{~cm}$ de comprimento padrão (LE), com diferenças entre machos e fêmeas. A maior TMCA das fêmeas (32,6 centímetros LE) e machos (32 cm LE) foi observada em 1980, nos anos seguintes este parâmetro variou entre 30 e 26,4 cm em fêmeas e entre 26 LE e 29 LE cm em machos. No ciclo anual as maiores TMCM das fêmeas foram observadas de abril a junho e em machos de maio e junho. O TMG de fêmeas foi significativamente maior em 1980 (33,5 centímetros LE), nos anos seguintes, variou entre 27,1 e 31,8 cm LE; em machos durante todo o período de estudo variou entre 25,8 e 29,5 centímetros LE. A população de $P$. marie baixa pressão de pesca na área de estudo, não é afetado por sobrepesca ao crescimento nem ao recrutamento.

Palabras chave: Pescaria fluvial, tamanhos de maturação gonadal, espécies migratórias, dinâmica populacional.

\section{Introducción}

Los peces detritívoros del género Prochilodus, son considerados el soporte que sustenta la pesquería de los grandes ríos suramericanos, debido a que juegan un papel primordial en la transferencia de energía en la cadena trófica, contribuyen al ciclaje de nutrientes, regulan el trasporte de carbono (Taylor et al., 2006; Mclntyre et al., 2007) y representan más del $50 \%$ de la biomasa de estos ecosistemas (Bayley y Petrere, 1989; Sverlij et al., 1993; Taylor et al., 2006). Según Castro y Vari (2004), el género Prochilodus incluye 13 especies, dentro de las cuales está el coporo, Prochilodus mariae, que presenta amplia distribución en la cuenca del Orinoco. Esta especie de agua dulce es la más explotada por las pesquerías de Colombia y Venezuela, se considera que en el año 2008, alcanzo una captura total de 8750 t (Machado-Allison y Bottini, 2010; Ramírez-Gil et al., 2011), sin embargo, aunque ha sido explotada por más de 50 años, no es una especie considerada amenazada en los libros rojos de Colombia y Venezuela.

Dada su importancia comercial y ecológica, se han conducido varios estudios que han permitido cono- cer sus parámetros de edad y crecimiento (Lilyestrom, 1983; Barbarino et al., 1998), aspectos reproductivos (Saldaña y Venables, 1981), hábitos alimenticios (Beltrán-Hostos et al., 2001), migraciones (Usma et al., 2013) y desembarcos en los puertos pesqueros de la Orinoquia colombiana (Ramírez-Gil et al., 2011) y de la Orinoquia venezolana (Novoa, 2002; Machado-Allison y Bottini, 2010).

Sin embargo no se ha realizado un análisis del comportamiento de las tallas de captura y de madurez sexual de esta especie en los ríos donde se lleva a cabo su pesquería en Colombia, aspecto importante de evaluar ya que la pesquería remueve de la población los individuos adultos de mayor talla y favorece individuos que ponen su energía para reproducirse temprano a menor tamaño (Stearns, 1992 citado en Uusi-Heikkil et al., 2015), lo cual puede afectar la población al reducir la fecundidad y el reclutamiento y aumentar la sensibilidad a cambios ambientales (Laugen et al., 2014) .

Adicional a la pesca factores ambientales como la ocurrencia de anomalías térmicas ENSO (El Niño Southern Oscillation), también pueden afectar las poblaciones de peces como ha sido señalado para Prochilodus 
magdalenae que no alcanza la madurez, ni las tallas mínimas de captura cuando se presentan estas anomalías encadenadas (Instituto de Hidrología, Meteorología y Estudios Ambientales - IDEAM, 2002). En la Orinoquia su ocurrencia ocasiona déficit de precipitación con la consecuente disminución del caudal de los ríos, que podría alterar los ciclos biológicos de las especies de peces.

En este documento se analiza el comportamiento de las tallas medias de captura anual relacionado con factores ambientales y el de las tallas medias de madurez gonadal, para establecer si hay afectación de la población objeto de presión de pesca de esta especie en la parte alta del río Meta.

\section{Materiales y métodos}

\section{Área de estudio}

La investigación se llevó a cabo en el área de influencia de Puerto López (Meta), puerto pesquero del río Metica, parte alta del río Meta (Figura 1).

\section{Fase de campo}

Los registros de tallas de captura de $P$. mariae fueron recopilados mediante muestreos a los desembarcos; de 1980 a 1986 se realizó un muestreo mensual y de 1987 a 2002 se incrementó el esfuerzo a cuatro monitoreos mensuales, la fecha de los muestreos se seleccionó de manera aleatoria; entre los años 2006 a 2011 el muestreo fue diario. Sin información para los años 2003-2005.

En cada muestreo, los ejemplares fueron medidos, longitud estándar (LE), con una cinta métrica $(0,001 \mathrm{~m}$ de aproximación), sexados y clasificado su estado de madurez gonadal macroscópicamente, de acuerdo con la escala que se presenta en la tabla 1, modificada de Bazigos (1976).

Los datos utilizados en este trabajo corresponden a la base de datos del Sistema de Información Sectorial Pesquero y Acuícola, suministrada por la Subgerencia de Pesca y Acuicultura del Instituto Nacional de Desarrollo Rural - INCODER, Sugerencia de Pesca del Instituto Colombiano Agropecuario - ICA y la Autoridad Nacional de Acuicultura y Pesca - AUNAP.

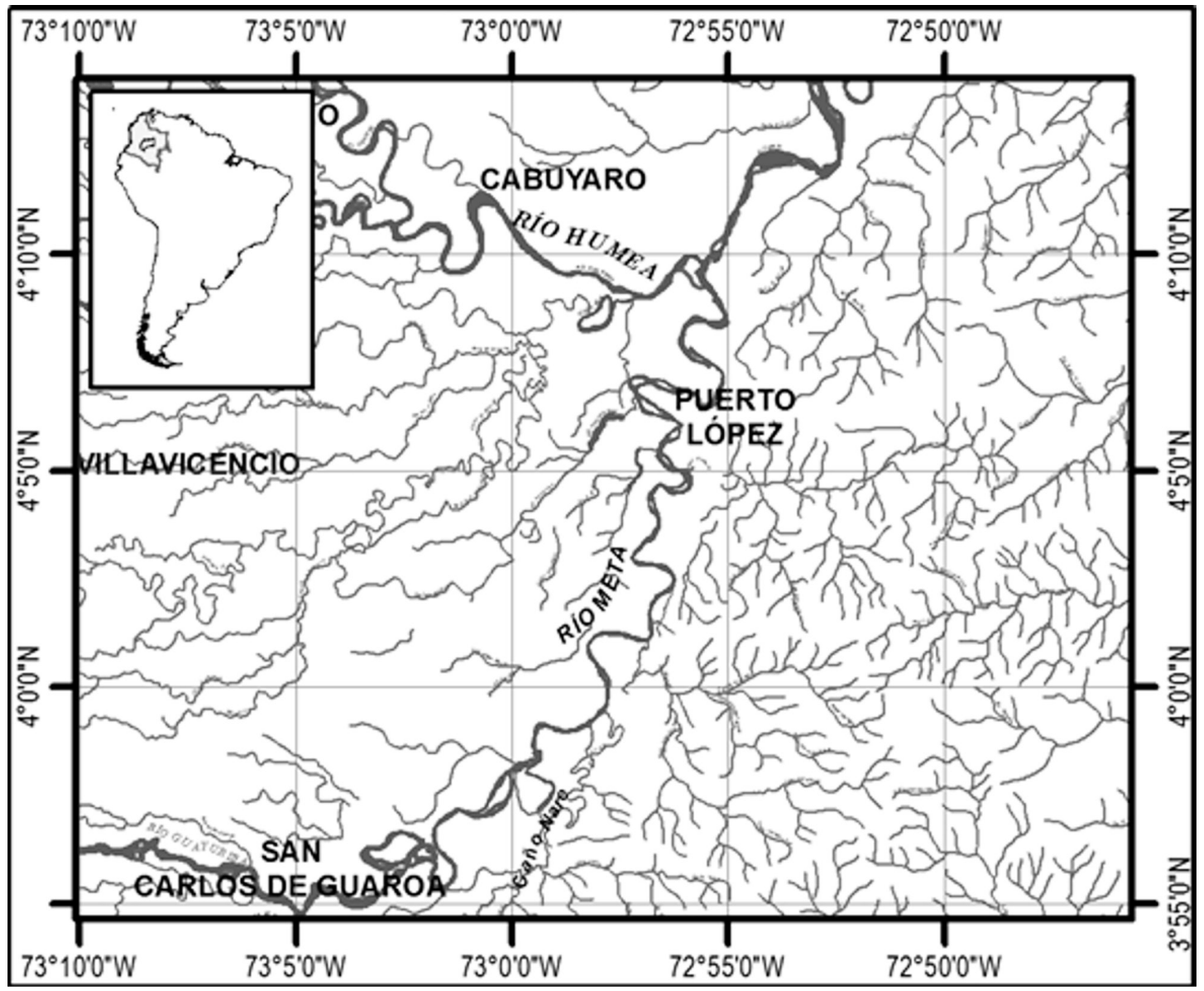

Figura 1. Localización del área de influencia de Puerto López (Meta). Cartografía base IGAC Escala 1:100000. 
Tabla 1. Estados de madurez gonadal

\begin{tabular}{|c|l|l|}
\hline Estado & \multicolumn{1}{|c|}{ Hembras } & \multicolumn{1}{c|}{ Machos } \\
\hline I & Ovarios transparentes & Testículos transparentes \\
\hline II & Ovarios vascularizado de color rosado & Testículo opaco \\
\hline III & Ovarios de color amarillento con óvulos visibles & Testículo de color rosado pálido \\
\hline IV & $\begin{array}{l}\text { Óvulos visibles, bien formados pero } \\
\text { no se desprenden del ovario }\end{array}$ & $\begin{array}{l}\text { Color blanco lechoso, pero no } \\
\text { fluye el esperma al presionar }\end{array}$ \\
\hline V & $\begin{array}{l}\text { Ovarios de color anaranjado, óvulos } \\
\text { grandes que al presionar un poco se } \\
\text { desprenden huevos. En este nivel de la } \\
\text { escala fueron incluidas las desovadas. }\end{array}$ & $\begin{array}{l}\text { Color blanco lechoso, desprende fácilmente } \\
\text { gotas de esperma. Se incluyen los machos } \\
\text { con testículos sanguinolentos y fláccidos, } \\
\text { que ya han liberado el esperma. }\end{array}$ \\
\hline
\end{tabular}

\section{Tallas de captura}

Los datos de tallas de captura (longitud estándar - LE, en $\mathrm{cm}$ ), se analizaron mediante la prueba t de Student para establecer si había diferencia significativa entre las tallas de las hembras y los machos. Con estadística descriptiva (promedio y desviación estándar), se calcularon las tallas medias de captura anual (TMCA) y mensual (TMCM) de los ciclos anuales estudiados, a partir de 13.444 registros.

Se realizaron pruebas de hipótesis para comparar los estimativos de TMCA entre ciclos hidrológicos y los de TMCM dentro del ciclo anual. Al conjunto de los datos se les aplicó la prueba Levene, para determinar si existía homogeneidad de varianza en los registros de tallas entre años y entre meses y como en ningún caso se cumplió el supuesto de normalidad se utilizaron las pruebas no paramétricas de Kruskall-Wallis y Mann-Whitney, con el paquete estadístico PAST V.2.06 (Hammer et al., 2001) con significancia $\mathrm{P}<0,05$.

Se utilizaron los modelos de regresión lineal, logarítmico, inverso, cuadrático, cúbico, potencia, compuesto, s cruzada, crecimiento y exponencial para establecer las posibles relaciones entre las tallas de captura, nivel del río y anomalías térmicas ENSO (El Niño Southern Oscillation) con significancia $\mathrm{P}<0,05$; para este análisis se utilizaron los estimativos de TMCM, promedio mensual de los niveles del río Metica (Estación Puente Lleras, suministrados por el IDEAM) y promedio mensual de las anomalías ENSO (Tomados de http://www.cpc.ncep.noaa. gov/products/precip/CWlink/MJO/enso.shtml\#history) de los años 1980 a 2011.

\section{Talla de madurez gonadal- TMG}

Para efectos del análisis se agruparon los datos de madurez en dos grupos: maduros e inmaduros; en el primero se incluyeron los estados IV y $\mathrm{V}$ y en el de inmaduros los estados I a III. Los datos de LE de machos y hembras maduros se compararon mediante la prueba estadística t de Student, para establecer si había diferencia significativa entre los registros de los dos sexos. De igual manera los registros de tallas de ejemplares maduros de hembras y de machos se compararon entre años, utilizando la prueba de Levene para establecer la homocedasticidad de varianza y como no se cumplieron los supuestos de normalidad se realizó la prueba de Kruskall-Wallis y Mann-Whitney para establecer si había diferencia entre años con significancia $\mathrm{P}<0,05$; se utilizó el programa Past V. 2.06 (Hammer et al., 2001).

La talla media de madurez gonadal para la especie se calculó a partir de 8.291 registros de hembras de los años 1980 a 2011 y de 5.050 de machos de los años 1983 a 2011; sin registros en los años 2003 a 2005 y 2008 para ambos sexos. Se establecieron 22 marcas de clase (entre 19 y $40 \mathrm{~cm} \mathrm{LE}$ ) y para cada una de ellas se estimaron la frecuencia absoluta, la frecuencia relativa y la frecuencia relativa acumulada.

La frecuencia relativa acumulada se linealizó (Tfra) mediante la ecuación:

$$
\text { Tfra }=\operatorname{Ln}\left(\frac{1}{\text { fra }}-1\right)
$$

Donde Ln es logaritmo natural y fra es frecuencia acumulada.

A partir de la regresión lineal entre la marca de clase y Tfra se estimaron los parámetros a y b de la curva logística:

$$
T M G=\left(1+e^{(a-(b+L E))}\right)^{-1}
$$

Las tallas de madurez a $25 \%, 50 \%$ y $75 \%$ para cada año se estimaron mediante las siguientes ecuaciones: 


$$
\begin{gathered}
T_{M G_{25}}=-\left[\frac{a-\operatorname{Ln}(3)}{b}\right] \\
T M G_{L_{50}}=\frac{a}{b} \\
T M G_{L_{75}}=-\left[\frac{a+\operatorname{Ln}(3)}{b}\right]
\end{gathered}
$$

Donde a y b son los parámetros de la curva logística y Ln(3) corresponde al logaritmo natural de 3.

\section{Resultados}

En el período de estudio, los registros de tallas de captura de $P$. mariae en Puerto López variaron entre 14 y $45 \mathrm{~cm} \mathrm{LE}$, con diferencia significativa $(P<0,05)$ entre hembras y machos, por lo que para el presente estudio se analizaron los dos sexos por separado.

\section{Dinámica de las tallas medias de captura entre ciclos anuales}

Las tallas de las hembras se registraron en un rango de 14 a 45 cm LE. Se encontró diferencia estadística $(\mathrm{P}<0,05)$ entre las TMCA de los años estudiados, con una ligera tendencia hacia la disminución. Así en el primer año de muestreo este parámetro fue significativamente mayor (32,6 cm LE) que en los años siguientes; posteriormente se ubicó por encima de los $30 \mathrm{~cm}$ LE en los años 1981, 1985, 1988 y 1989 y en los años restantes, esta medida osciló entre 29,8 y 26,4cm LE (Figura 2).

El rango de tallas de los machos varió entre 16 y $42 \mathrm{~cm}$ LE. De manera similar a lo registrado con las hembras, también se observó diferencia significativa $(P<0,05)$ en la TMCA entre años, con tendencia hacia la disminución. En el primer año de muestreo este parámetro se situó por encima de los $32 \mathrm{~cm}$ de LE, valor significativamente mayor $(\mathrm{P}<0.05)$ al reportado para los años posteriores (entre 26 y 29 cm LE) (Figura 3).

No se encontró ninguna relación entre las TMCM de hembras y machos con el nivel del río Metica y las anomalías térmicas (ENSO).

\section{Estructura de tallas en el ciclo anual}

Dentro del ciclo hidrológico, las TMCM de las hembras variaron significativamente entre meses $(P<0,05)$; la menor talla promedio (28 cm LE), se estimó en los meses de noviembre a febrero (período de aguas descendentes y bajas); a partir de marzo se incrementó hasta llegar a $31 \mathrm{~cm}$ LE en mayo, valor significativamente más alto comparado con los demás meses $(\mathrm{P}<0,05)$. A partir de este mes descienden hasta $29 \mathrm{~cm}$ nivel en el que permaneció de julio a octubre, para iniciar de nuevo el ciclo en noviembre (Figura 4).

En los machos el comportamiento de las TMCM fue similar al de las hembras; la menor talla (27 cm LE) se presentó en los meses de noviembre a marzo (aguas

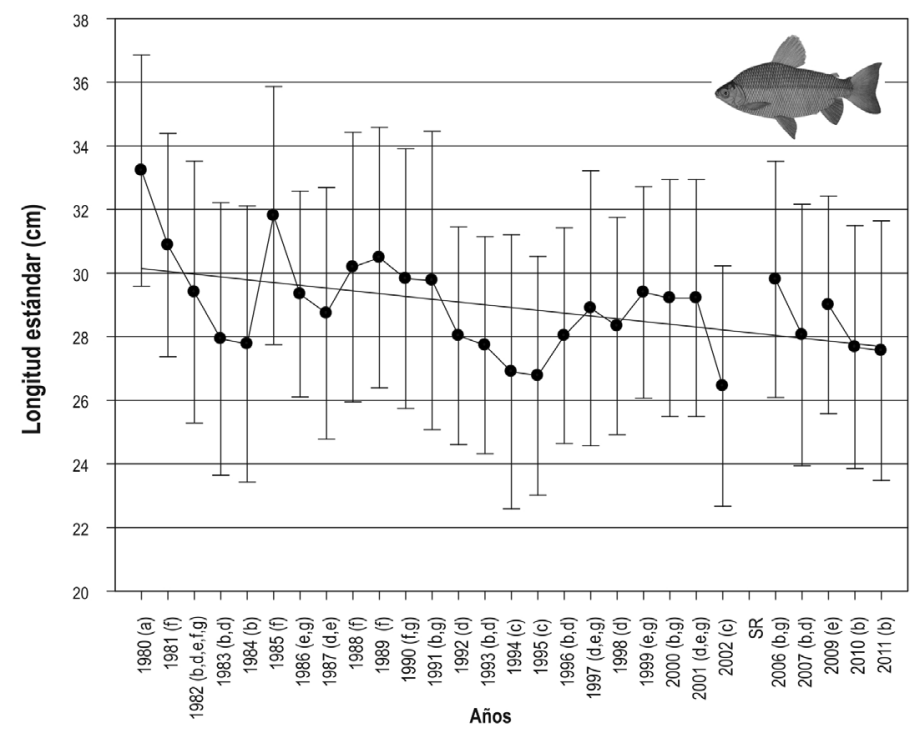

Figura 2. Tallas medias de captura anual de hembras, en el puerto pesquero de Puerto López en los años 1980 a 2011 n=8.291. Letras iguales indican sin diferencia significativa $(\mathrm{P}<0,05)$ 


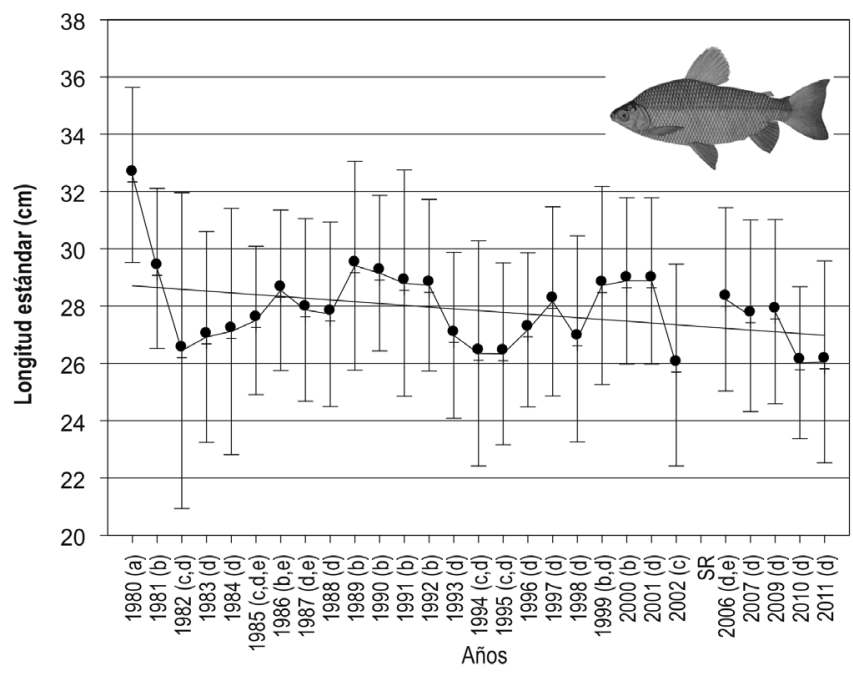

Figura 3. Tallas medias de captura anual de machos, en el puerto pesquero de Puerto López en los años 1980 a 2011 . Letras iguales indican sin diferencia significativa $(P<0,05)$ en la TMCA. $n=5.153$

descendentes y bajas), siendo significativamente diferentes de los otros meses $(\mathrm{P}<0,05)$ y las mayores $(28 \mathrm{a}$ $29 \mathrm{~cm}$ LE) se observaron en el período de abril a junio (aguas ascendentes), superiores a las del resto del año $(\mathrm{P}<0,05)$ (Figura 5).

\section{Comparación histórica de las tallas de madurez gonadal en la parte alta del río Meta}

Se encontró diferencia significativa entre los registros de machos y hembras $(\mathrm{P}<0,05)$ utilizados para la esti- mación de las tallas de madurez gonadal, por lo anterior se presentan los resultados separados por sexo.

El rango de tallas de hembras maduras osciló entre 18 y 45 cm LE. Los estimativos de TMG L25, L50, L75, se presentan en la figura 6 y los parámetros de la curva logística en la tabla 2. Al analizar los estimativos de TMGL50 de hembras se encontró que este parámetro en el año 1980 (33, 5 cm LE) fue significativamente más alto que el estimado para los años siguientes, en los que varió entre 27,1 y $31,8 \mathrm{~cm} \mathrm{LE}$, pero sin que se pudiera establecer alguna tendencia a través del tiempo.

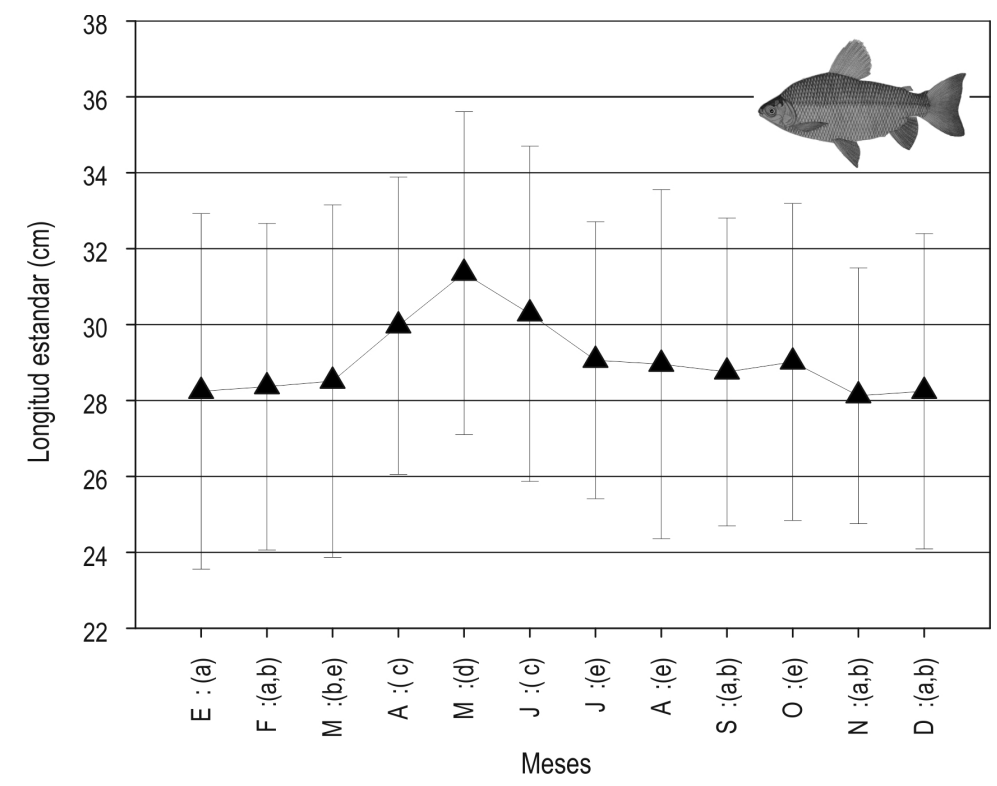

Figura 4. Comportamiento de la talla media de captura mensual (TMCM) de las hembras de P. mariae en Puerto López a lo largo del ciclo hidrológico (promedios mensuales de 28 años). Letras iguales indican sin diferencia significativa $(P<0,05)$. 


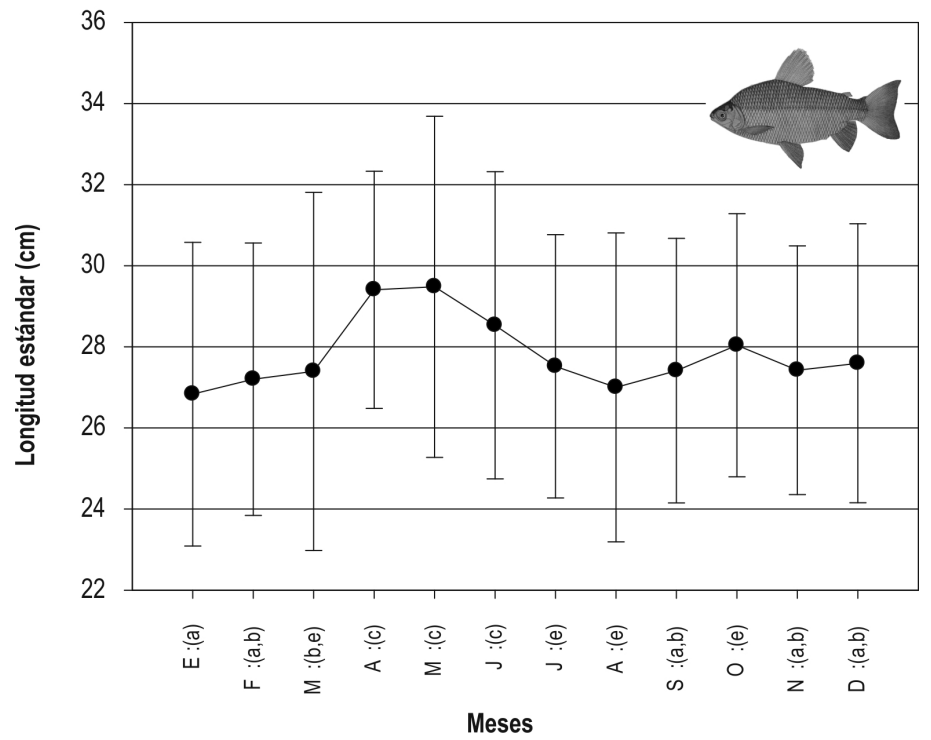

Figura 5. Comportamiento de la talla media de captura mensual (TMCM) de los machos de P. mariae en Puerto López a lo largo del ciclo hidrológico (promedios mensuales de 28 años). Letras iguales indican sin diferencia significativa $(P<0,05)$.

Se reportaron machos maduros con tallas desde los $16 \mathrm{~cm}$ LE hasta los $42 \mathrm{~cm}$ LE. Los estimativos de la TMG L25, L50, L75, para los años analizados, se presentan en la figura 7 y los parámetros de la curva logística en las tablas 2 y 3 . Se observó diferencia significativa en la TMG L50, entre los diferentes años $(\mathrm{P}<0,05)$, sin presentar una tendencia definida a lo largo del tiempo.

\section{Discusión}

\section{Dinámica de las tallas medias de captura entre ciclos anuales}

Las mayores TMCA, tanto de hembras como de machos, se registraron en el año 1980, siendo significativamente diferentes a las de los años restantes; situación que es normal al inicio de una pesquería,

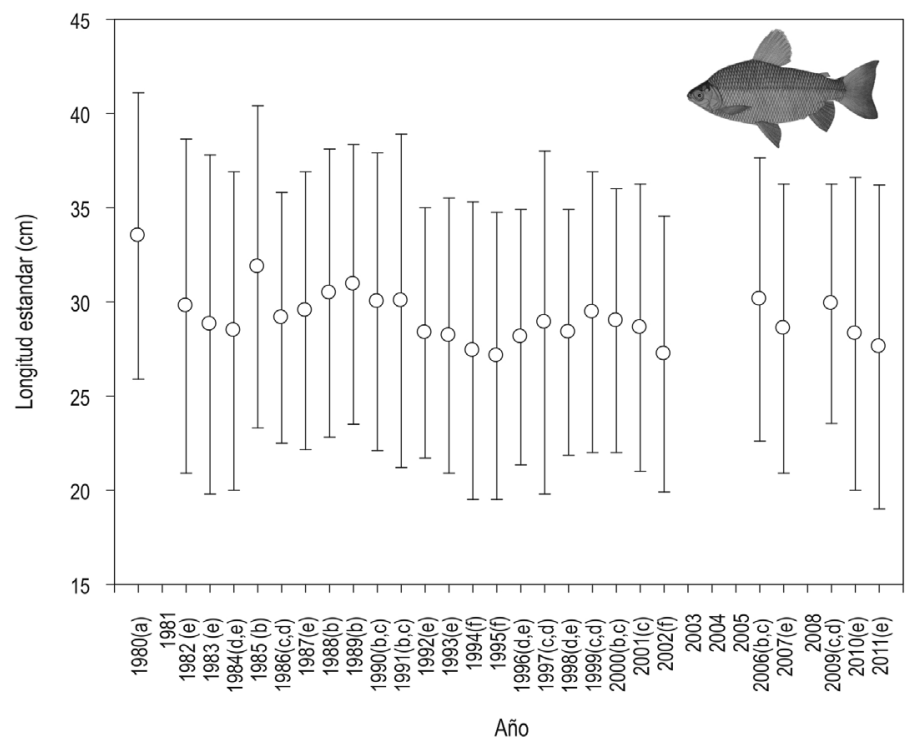

Figura 6. Tallas madurez gonadal L25, L50, L75 de las hembras de $P$. mariae en la parte alta del río Meta. Letras iguales indican sin diferencia significativa $(P<0,05)$. 
Tabla 2. Parámetros a y b de la regresión entre la frecuencia acumulada y la longitud estándar en hembras sexualmente maduras de P. mariae con los límites de confianza a 95\% y su correlación en los años 1980 a 2011.

\begin{tabular}{|c|c|c|c|c|c|c|c|c|}
\hline \multirow{2}{*}{$\begin{array}{l}\text { Año } \\
1980\end{array}$} & \multirow{2}{*}{$\begin{array}{l}\mathbf{n} \\
85\end{array}$} & \multirow{2}{*}{$\begin{array}{c}\mathbf{a} \\
16,167\end{array}$} & \multicolumn{2}{|c|}{$\begin{array}{l}\text { Límites de confianza } \\
\text { a }\end{array}$} & \multirow{2}{*}{$\begin{array}{c}\mathbf{b} \\
-0,482\end{array}$} & \multicolumn{2}{|c|}{$\begin{array}{l}\text { Límites de confianza } \\
\text { b }\end{array}$} & \multirow{2}{*}{$\begin{array}{c}\mathbf{R}^{2} \\
0,987\end{array}$} \\
\hline & & & 15,253 & 17,081 & & $-0,509$ & $-0,456$ & \\
\hline 1982 & 25 & 12,310 & 10,813 & 13,808 & $-0,414$ & $-0,464$ & $-0,363$ & 0,957 \\
\hline 1983 & 113 & 11,673 & 9,918 & 10,813 & $-0,405$ & $-0,460$ & $-0,350$ & 0,931 \\
\hline 1984 & 206 & 12,346 & 10,920 & 13,772 & $-0,433$ & $-0,480$ & $-0,387$ & 0,952 \\
\hline 1985 & 41 & 13,662 & 12,299 & 15,025 & $-0,429$ & $-0,470$ & $-0,387$ & 0,973 \\
\hline 1986 & 103 & 16,063 & 14,046 & 18,080 & $-0,551$ & $-0,619$ & $-0,484$ & 0,964 \\
\hline 1987 & 482 & 14,620 & 13,307 & 15,934 & $-0,495$ & $-0,536$ & $-0,454$ & 0,970 \\
\hline 1988 & 360 & 14,625 & 13,395 & 15,854 & $-0,480$ & $-0,519$ & $-0,440$ & 0,970 \\
\hline 1989 & 285 & 15,289 & 14,269 & 16,309 & $-0,494$ & $-0,527$ & $-0,461$ & 0,980 \\
\hline 1990 & 264 & 13,931 & 13,652 & 14,210 & $-0,464$ & $-0,473$ & $-0,455$ & 0,998 \\
\hline 1991 & 363 & 12,464 & 11,762 & 13,167 & $-0,415$ & $-0,437$ & $-0,393$ & 0,986 \\
\hline 1992 & 128 & 15,554 & 14,036 & 17,071 & $-0,549$ & $-0,599$ & $-0,499$ & 0,974 \\
\hline 1993 & 249 & 14,176 & 13,140 & 15,212 & $-0,503$ & $-0,534$ & $-0,469$ & 0,981 \\
\hline 1994 & 357 & 12,747 & 11,218 & 14,276 & $-0,465$ & $-0,516$ & $-0,414$ & 0,954 \\
\hline 1995 & 582 & 13,008 & 12,390 & 13,626 & $-0,480$ & $-0,500$ & $-0,459$ & 0,992 \\
\hline 1996 & 95 & 15,204 & 14,450 & 15,958 & $-0,541$ & $-0,566$ & $-0,515$ & 0,993 \\
\hline 1997 & 143 & 11,646 & 10,640 & 12,652 & $-0,403$ & $-0,435$ & $-0,371$ & 0,977 \\
\hline 1998 & 201 & 15,966 & 14,655 & 17,277 & $-0,563$ & $-0,608$ & $-0,518$ & 0,980 \\
\hline 1999 & 74 & 14,545 & 13,201 & 15,889 & $-0,494$ & $-0,537$ & $-0,451$ & 0,980 \\
\hline 2000 & 312 & 15,163 & 13,922 & 16,403 & $-0,523$ & $-0,566$ & $-0,481$ & 0,976 \\
\hline 2001 & 190 & 13,733 & 12,162 & 15,305 & $-0,480$ & $-0,532$ & $-0,427$ & 0,959 \\
\hline 2002 & 271 & 13,573 & 12,160 & 14,987 & $-0,499$ & $-0,546$ & $-0,452$ & 0,962 \\
\hline 2006 & 712 & 14,648 & 14,212 & 15,084 & $-0,486$ & $-0,500$ & $-0,473$ & 0,996 \\
\hline 2007 & 237 & 13,630 & 12,079 & 15,181 & $-0,477$ & $-0,529$ & $-0,425$ & 0,955 \\
\hline 2008 & 44 & 12,394 & 10,998 & 13,791 & $-0,366$ & $-0,409$ & $-0,322$ & 0,933 \\
\hline 2009 & 458 & 17,240 & 15,755 & 18,725 & $-0,576$ & $-0,624$ & $-0,529$ & 0,970 \\
\hline 2010 & 683 & 12,485 & 11,570 & 13,400 & $-0,441$ & $-0,469$ & $-0,413$ & 0,979 \\
\hline 2011 & 1158 & 11,747 & 11,340 & 12,155 & $-0,426$ & $-0,438$ & $-0,413$ & 0,995 \\
\hline
\end{tabular}




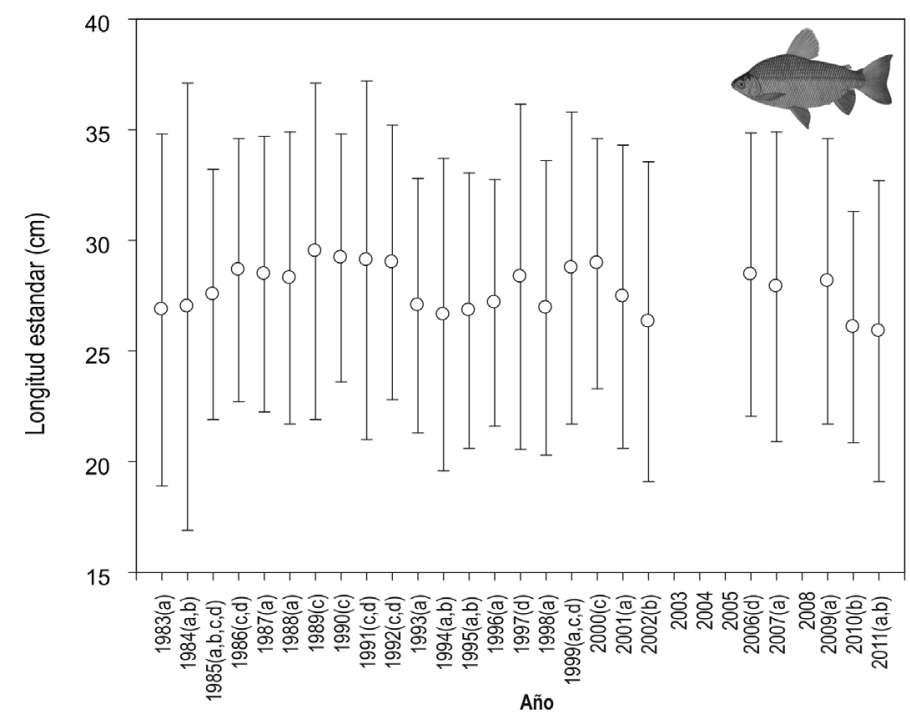

Figura 7. Tallas medias de madurez gonadal de los machos de $P$. mariae en la parte alta del río Meta. Letras iguales indican sin diferencia significativa $\mathrm{P}<0,05$.

cuando se capturan los ejemplares más longevos y de mayor talla, comportamiento atribuido a la mortalidad por pesca (Ricker, 1981; Daan et al., 2005; Hutchings, 2005; Shin et al., 2005; Wheeler et al., 2009).

Sin embargo, las variaciones anuales posteriores, no se explican solo por este factor, ya que las tallas también pueden ser influenciadas por aspectos ambientales (Blanco et al., 2007; Cheung et al., 2013); sin embargo en los análisis realizados en este estudio no se encontró correlación entre las tallas y los parámetros ambientales como cambios en el nivel del río o en las anomalías ENSO de los años estudiados. Esto lleva a pensar que la variación en las TMCA se presentan como resultado de un mayor número de factores, a los contemplados en el presente estudio, tales como variaciones interanuales del reclutamiento (Suzuki et al., 2009), introducción de nuevos artes de pesca y modificaciones en el diámetro del ojo de malla, que afectan la distribución de tallas de captura (Sparre y Venema 1997), o variaciones entre sitios, temporadas y artes de pesca que se ven reflejadas en los tamaños de captura de las especies (Narváez et al., 2008).

\section{Estructura de tallas en el ciclo anual}

De acuerdo con los resultados obtenidos claramente se observó variación estacional en el ciclo anual de las TMCM; este comportamiento se explica por la etología de la especie que se caracteriza por ser migratoria (Barbarino et al., 1998; Usma et al., 2009, Usma et al., 2013), característica que comparte con otras especies del mismo género como $P$. affinis (Sato et al., 1996), P. argenteus (Godinho y Kynard, 2006), P. brevis (De Lima et al., 2012), P. costatus (Melo et al., 2013), P. magdalenae (Jaramillo-Villa y Jímenez-Segura, 2008), P. linneatus (Copeleti y Petrere, 2006; Pesoa y Shulz, 2010) y P. nigricans (Anderson et al., 2009).

En el período de aguas descendentes y bajas (noviembre a febrero), cuando las áreas de rebalse se secan y las lagunas reducen su tamaño, los juveniles abandonan esas zonas y se concentran en el canal principal (Beltrán Hostos et al., 2001; Ramírez-Gil et al., 2011), donde proporcionalmente son más abundantes que los adultos, explicando las bajas tallas de captura en ese periodo.

En el período de aguas ascendentes, la población parental migra hacia las partes altas del río a reproducirse (Barbarino et al., 1998; Beltrán-Hostos et al., 2001, Ramírez-Gil et al., 2011), y la presión de pesca se dirige hacia ese grupo, lo que se refleja en un aumento en la TMCM en abril, mayo y junio. En los meses restantes la pesca se concentra en los rebalses y áreas inundadas, donde se capturan ejemplares de tamaño intermedio.

\section{Dinámica histórica de la talla de madurez gonadal en la parte alta del río Meta}

Similar a lo observado con la TMCA, la TMG L50 de las hembras en 1980 fue significativamente mayor a la del resto de los años estudiados, confirmando que 
Tabla 3. Parámetros a y b de la regresión entre la fre cuencia acumulada y la longitud estándar en machos sexualmente maduros de P. mariae con los límites de confianza a 95\% y correlación en los años 1983 a 2011.

\begin{tabular}{|c|c|c|c|c|c|c|c|c|}
\hline \multirow{2}{*}{$\begin{array}{l}\text { Año } \\
1983\end{array}$} & \multirow{2}{*}{$\begin{array}{l}\mathbf{n} \\
52\end{array}$} & \multirow{2}{*}{$\begin{array}{c}\mathbf{A} \\
12,375\end{array}$} & \multicolumn{2}{|c|}{ Límites de confianza a } & \multirow{2}{*}{$\begin{array}{c}\mathbf{b} \\
-0,460\end{array}$} & \multicolumn{2}{|c|}{$\begin{array}{l}\text { Límites de confianza } \\
\text { b }\end{array}$} & \multirow{2}{*}{$\begin{array}{c}\mathbf{R}^{\mathbf{2}} \\
0,971\end{array}$} \\
\hline & & & 11,023 & 13,726 & & $-0,508$ & $-0,413$ & \\
\hline 1984 & 70 & 9,763 & 8,541 & 10,984 & $-0,361$ & $-0,401$ & $-0,322$ & 0,954 \\
\hline 1985 & 12 & 17,879 & 13,195 & 22,564 & $-0,649$ & $-0,815$ & $-0,482$ & 0,928 \\
\hline 1986 & 38 & 17,582 & 16,096 & 19,067 & $-0,614$ & $-0,664$ & $-0,564$ & 0,984 \\
\hline 1987 & 221 & 16,799 & 15,369 & 18,228 & $-0,59$ & $-0,638$ & $-0,543$ & 0,974 \\
\hline 1988 & 149 & 15,661 & 13,946 & 17,376 & $-0,554$ & $-0,611$ & $-0,496$ & 0,964 \\
\hline 1989 & 61 & 14,169 & 13,306 & 15,033 & $-0,48$ & $-0,509$ & $-0,451$ & 0,989 \\
\hline 1990 & 60 & 19,088 & 18,319 & 19,858 & $-0,653$ & $-0,679$ & $-0,628$ & 0,996 \\
\hline 1991 & 84 & 13,144 & 12,369 & 13,918 & $-0,452$ & $-0,477$ & $-0,427$ & 0,987 \\
\hline 1992 & 55 & 17,095 & 14,436 & 19,753 & $-0,59$ & $-0,682$ & $-0,497$ & 0,954 \\
\hline 1993 & 92 & 17,252 & 15,726 & 18,777 & $-0,637$ & $-0,690$ & $-0,584$ & 0,983 \\
\hline 1994 & 217 & 13,774 & 11,719 & 15,829 & $-0,517$ & $-0,590$ & $-0,445$ & 0,940 \\
\hline 1995 & 305 & 15,755 & 14,332 & 17,179 & $-0,587$ & $-0,636$ & $-0,538$ & 0,976 \\
\hline 1996 & 88 & 17,909 & 16,969 & 18,849 & $-0,659$ & $-0,692$ & $-0,625$ & 0,994 \\
\hline 1997 & 342 & 13,316 & 12,264 & 14,368 & $-0,470$ & $-0,504$ & $-0,435$ & 0,977 \\
\hline 1998 & 68 & 13,958 & 12,832 & 15,084 & $-0,516$ & $-0,555$ & $-0,477$ & 0,980 \\
\hline 1999 & 18 & 14,819 & 12,837 & 16,801 & $-0,515$ & $-0,583$ & $-0,447$ & 0,963 \\
\hline 2000 & 126 & 18,791 & 17,547 & 20,035 & $-0,649$ & $-0,692$ & $-0,605$ & 0,989 \\
\hline 2001 & 186 & 14,721 & 13,991 & 15,451 & $-0,536$ & $-0,561$ & $-0,511$ & 0,992 \\
\hline 2002 & 184 & 13,307 & 12,23 & 14,384 & $-0,506$ & $-0,543$ & $-0,468$ & 0,981 \\
\hline 2006 & 548 & 16,319 & 15,768 & 16,871 & $-0,574$ & $-0,591$ & $-0,556$ & 0,996 \\
\hline 2007 & 302 & 14,594 & 13,795 & 15,393 & $-0,523$ & $-0,549$ & $-0,496$ & 0,989 \\
\hline 2008 & 27 & 21,925 & 18,764 & 25,087 & $-0,632$ & $-0,722$ & $-0,542$ & 0,967 \\
\hline 2009 & 410 & 16,028 & 15,006 & 17,050 & $-0,569$ & $-0,605$ & $-0,534$ & 0,987 \\
\hline 2010 & 679 & 18,245 & 17,455 & 19,035 & $-0,700$ & $-0,728$ & $-0,672$ & 0,995 \\
\hline 2011 & 899 & 13,973 & 13,286 & 14,660 & $-0,540$ & $-0,563$ & $-0,516$ & 0,993 \\
\hline
\end{tabular}

al inicio de la pesquería se capturaban los ejemplares de mayor talla y más longevos como se anotó anteriormente.

Posteriormente el valor de la TMG L50, en las hembras y en los machos, osciló entre los años, sin ninguna tendencia; comportamiento que también se ha observado en especies marinas migratorias como Trachurus murphyi en el análisis realizado con datos de tallas de madurez de 1967 a 2012 (Perea et al., 2013). Si bien la literatura reporta que la presión de pesca induce hacia la disminución de la talla de maduración de las po- blaciones explotadas (Jørgensen et al., 2007; Conover, 2009, Sharpe y Hendry, 2009, Audzijonyte et al., 2013; Uusi-Heikkilä et al., 2015), esto no se evidenció en P. mariae en el alto Meta, en el período de 1980 a 2011.

Al comparar año a año la TMCA con la TMG L50, la diferencia observada entre estos dos parámetros fue menor a $3 \mathrm{~mm}$ en promedio, lo que indicó que a lo largo del tiempo, sobre la población de $P$. mariae en el área de estudio, no se presentó sobrepesca al crecimiento. 
El análisis de las tallas de $P$. marie no muestra evidencia de afectación en la población bajo presión de pesca; es posible que esto se deba a que la especie es capturada con redes de ahorque cuya selectividad tiene forma de campana, permitiendo el escape de peces por encima y por debajo del rango de captura, con lo que se logra mantener la variabilidad genética y la estructura de tallas de la población a más largo plazo (Jørgensen et al., 2009).

Se considera pertinente y necesario mantener la veda en su período reproductivo a fin de evitar la sobrepesca al reclutamiento y extremar el cuidado de los humedales que le sirven de refugio a larvas y alevinos, ya que las fases de pre-reclutamiento están afectadas por factores como disponibilidad de alimento y depredación (Cadima 2003). Igualmente se recomienda el seguimiento a las tallas de desembarco y de madurez gonadal, para prever posibles alteraciones a la estructura poblacional a futuro.

\section{Agradecimientos}

Los autores agradecen a las entidades financiadoras del proyecto a partir del cual se originó el presente artículo: Universidad de los Llanos, Corporación Colombia Internacional-CCl, Instituto Nacional de Desarrollo Rural-INCODER, Autoridad Nacional de Acuicultura y Pesca-AUNAP y Fundación Nakuani.

\section{Referencias}

Anderson E, Montoya M, Soto A, Flores H. 2009. Challenges and Opportunities for Co-Management of a Migratory Fish (Prochilodus nigricans) in the Peruvian Amazon. American Fisheries Society Symposium, 69:741-756.

Audzijonyte A, Kuparinen A, Fulton EA. 2013. How fast is fisheriesinduced evolution? Quantitative analysis of modelling and empirical studies. Evolutionary Applications. DOI:10.1111/eva.12044

Barbarino A, Taphorn D, Winemiller K. 1998. Ecology of the coporo, Prochilodus mariae (Characiformes, Prochilodontidae), and status of annual migrations in western Venezuela. Environmental Biology of Fishes, 53:33-46.

Bayley PB, Petrere M. 1989. Amazon fisheries assessment of methods, status and management options. En: Dodge DP. (Editores). Proceedings of the international large river symposium. Canadian Special Publication of Fisheries and Aquatic Sciences 106, Ottawa. p 385-398.

Bazigos G. 1976. Estadísticas aplicadas a la pesca. FAO. Documento técnico de pesca N 135. Roma, FAO. p 181.

Beltrán-Hostos D, Ajiaco-Martínez RE y Ramírez-Gil H. 2001. Prochilodus mariae Eigenmann, 1922. En: Ramírez-Gil H, Ajiaco-Martínez RE (Editores.). La pesca en la baja Orinoquia colombiana: una visión integral. INPA. Bogotá-Colombia. p 96-99.
Blanco JR, Narváez BJC, Herrera PFA. ENSO and the rise and fall of a tilapia fishery in northern Colombia. Fisheries Research; 88:100-108

Cadima EL. 2003. Manual de evaluación de recursos pesqueros. FAO Documento Técnico de Pesca. No. 393. Roma, FAO. p 162.

Castro RMC. Vari rp. 2004. Detritivores of the South American fish family Prochilodontidae (Teleostei:Ostariophysi:Characiform es): A phylogenetic and revisionary study. Smithsonian Contributios to Zoology (SCZ), (622):1-189.

Cheung WWL, Sarmiento JL, Dunne J, Frolicher TL, Lam VWY, Deng ML, Watson R, Pauly D. 2013. Shrinking of fishes exacerbates impacts of global ocean changes on marine ecosystems. Nature Climte Change, 3:254-258.

Conover D. Munch SB, Arnott SA. 2009. Reversal of evolutionary downsizing caused by selective harvest of large fish. Proceedings of the Royal Society B. 276:2015-2020. DOI:10.1098/ rspb.2009.003

Copeleti AR, Petrere M Jr. 2006. Migration of the curimbatá Prochilodus lineatus (Valenciennes, 1836) (Pisces, Prochilodontidae) at the waterfall "Cachoeira de Em as" of the Mogi-Guaçu River - São Paulo, Brazil. Brazilian Journal of Biology, 66(2B): 651-659.

Daan N, Gislason H, Pope JG, Rice JC. 2005. Changes in the North Sea fish community: evidence of indirect effects of fishing? ICES Journal of Marine Science, 62:177-188

De Lima L,Verani JR, Chellappa S. 2012. Reproductive Ecology of Prochilodus brevis an Endemic Fish from the Semiarid Region of Brazil. The Scientific World Journal. DOI:10.1100/2012/810532

Godinho A, Kynard B. 2006. Migration and Spawning of RadioTagged Zulega Prochilodus argenteus in a Dammed Brazilian River. Transactions of the American Fisheries Society, 135:811824. DOI: 10.1577/T04-176.1

Hammer Ø, Harper DAT, Ryan PD. 2001. Past: Paleontological Statistics Software Package for Education and Data Analysis. Paleontologia Electronica, 4(1):9

Hutchings JA. 2005. Life history consequences of over-exploitation to population recovery in Northwest Atlantic cod (Gadus morhua). Canadian Journal of Fisheries and Aquatic Sciences, 62:824-832.

Jaramillo-Villa U, Jiménez-Segura F. 2008. Algunos aspectos biológicos de la población de Prochilodus magdalenae en las ciénagas de Tumaradó (Río Atrato), Colombia. Actualidades Biológicas, 30 (88): 55-66.

Jørgensen C, Enberg K, Dunlop ES, Arlinghaus R, Boukal DS, Brander $\mathrm{K}$, Ernande B et al. 2007. Managing the world's evolving fish stocks. Science 318:1247-1248.

Jørgensen C, Ernande B, Fiksen $\varnothing$, 2009. Size-selective fishing gear and life history evolution in the Northeast Artic cod. Evolutionary Applications. DOI:10.1111/j.1752-4571.2009.00075.x

Laugen AT, Engelhard GH, Whitlock R, Arlinghaus R, Dankel DJ, Dunlop ES et al. 2014. Evolutionary impact assessment:accounting for evolutionay consequences of fishing in an ecosystem approach to fisheries management. Fish Fish:15:65-96 
Lilyestrom CG. 1983. Aspectos de la biología del coporo Prochilodus mariae. Revista UNILLEZ de Ciencia y Tecnología, 1:5-11.

Machado-Allison A, Bottini B. 2010. Especies de la pesquería continental venezolana: un recurso natural en peligro. Boletín de la Academia de Ciencias Físicas, Matemáticas y Naturales de Venezuela, 70 (1):59-75.

McIntyre PB, Jones LE, Flecker AS, Vanni MJ. 2007. Fish extinctions alter nutrient recycling in tropical freshwaters. Proceedings of the National Academy of Sciences of the USA, 104:4461-6.

Melo B, Sato Y, Foresti F, Oliveir C. 2013. The roles of marginal lagoons in the maintenance of genetic diversity in the Brazilian migratory fishes Prochilodus argenteus and P. costatus. Neotropical Ichthyology, 11(3):625-636.

Narváez BJC, Herrera PFA, Blanco J. 2008. Efecto de los artes de pesca sobre el tamaño de los peces en una pesquería artesanal del Caribe colombiano. Boletín de Investigaciones Marinas y Costeras, 37 (2): 163-187.

Novoa, D. 2002. Los recursos pesqueros del eje fluvial Orinoco Apure: Presente y Futuro. INAPESCA. Caracas. p 148.

Perea AJ, Mori J, Buitrago B, Sánchez J. 2013. Aspectos reproductivos del jurel Trachurus murphyi en el Perú. En: Csirke J, Guevara-Carrasco R, Espino M. (Editores). Ecología, pesquería y conservación del jurel (Trachurus murphyi) en el Perú. Revista Peruana de Biología Número Especial 20(1):029-034.

Pesoa NA,Schulz UH. 2010. Diel and seasonal movements of grumatã Prochilodus lineatus (Valenciennes 1836) (Characiformes: Prochilodontidae) in the Sinos River, Southern Brazil. Brazilian Journal of Biology, 70(4):1169-1177.

Ramírez-Gil H, Ajiaco-Martínez RE, Barreto C, Valderrama M. 2011. Prochilodus mariae (Prochilodontidae). En: Lasso CA, Agudelo-Córdoba E, Jiménez-Segura LF, Ramírez-Gil H, Morales-Betancourt M, Ajiaco-Martínez RE, Gutiérrez FP, Usma JS, Muñoz-Torres SE, Sanabria- Ochoa AI (Editores). I. Catálogo de los recursos pesqueros continentales de Colombia. Serie Editorial Recursos Hidrobiológicos y Pesqueros Continentales de Colombia. Instituto de Investigación de los Recursos Biológicos Alexander von Humboldt (IAvH). Bogotá, D. C., Colombia. $p$ 312-316.

Ricker WE. 1981. Changes in the average size and average age of Pacific salmon. Canadian. Journal od Fisheries and Aquatic Sciences, 38:1636-1556

Saldaña JR, Venables BJ. 1981. Desarrollo sexual del coporo Prochilodus mariae (Prochilodontidae) en el rio Orinoco. Boletín del Instituto Oceanográfico de Venezuela, 1(2): 3-10.

Sato Y, Cardoso EL, Godinho AL, Godinho HP. 1996. Hypophysation of the fish Prochilodus affinis from the Rio Sao Francisco basin, Brazil. Arquivo Brasileiro de Medicina Veterinaria y Zootecnia, 48 (1):55-62

Sharpe D, Hendry A. 2009. Life history change in commercially exploited fish stocks: an analysis of trends across studies. Evolutionary Applications 2:260-275.

Shin YJ, Rochet MJ, Jennings S, Field J G, Gislason H. 2005. Using size-based indicators to evaluate the ecosystem effects of fishing. ICES Journal of Marine Science, 62: 384 - 396.

Sparre P, Venema SC. 1997. Introducción a la evaluación de recursos pesqueros tropicales. Parte 1. Manual. FAO Documento Técnico de Pesca. №. 306.1 Rev. 2. p 420.

Suzuki HI, Agostinho AA, Bailly D, Gimenes MF, Júlio-Junior HF, Gomes LC. 2009. Inter-annual variatiosn in the abundance of young-of-the year of migratory fishes in the Upper Paraná River floodplain: relations with hydrographic attributes. Brazilian Journal of Biology, 69 (2):649-660 (suplemento).

Sverlij SB, Espinach A, Ort’ G. 1993. Sinopsis de los datos biológicos y pesqueros del sábalo Prochilodus lineatus (Valenciennes 1847). FAO-FIR S154:1-64.

Taylor B, Flecker AS, Hall RO. 2006. Loss of a harvested fish species disrupts carbon flow in a diverse tropical river. Science, 313: 833-836.

Usma JS, Valderrama M, Escobar MD, Ajiaco-Martínez RE, VillaNavarro F, Castro F, Ramírez-Gil H, Sanabria Al, Ortega-Lara A, Maldonado-Ocampo J, Alonso JC, Cipamocha C. 2009. Peces dulceacuícolas migratorios en Colombia. En: Amaya JD, Naranjo L. (Editores.). Plan Nacional de las Especies Migratorias: Diagnóstico e identificación de acciones para la conservación y el manejo sostenible de las especies migratorias de la biodiversidad en Colombia. MAVDT- WWF. Bogotá D. C. Colombia. p 103-131.

Usma JS, Villa-Navarro F, Lasso CA, Castro F, Zuñiga-Upegui PT, Cipamocha C, Ortega-Lara A, Ajiaco-Martínez RE, Ramírez-Gil H, Jiménez LF, Maldonado-Ocampo J, Muñoz JA, Suárez JT. 2013. Peces dulceacuícolas migratorios de Colombia. En: Zapata LA, Usma JS. (Editores) Guía de las especies migratorias de la biodiversidad en Colombia. Peces. Vol 2. Ministerio de Ambiente y Desarrollo Sostenible/WWF-Colombia. Bogotá, D.C. Colombia. p 215-442

Uusi-Heikkilä S, Whiteley A, Kuparinen A, Matsumara S, VentureIli P, Wolter C, Slate J, Primmer C et al. 2015. The evolutionary legacy of size-selective harvesting extends from genes to populations. Evolutionary Applications. DOI:10.1111/ eva. 12268

Wheeler JP, Purchase CF, Macdonald PDM, Fill R, Jacks L, Wang $\mathrm{H}, \mathrm{Ye}$ C. 2009. Temporal changes in maturation, mean lenghtat-age, and condition of spring-spawning Atlantic herring (Clupea harengus) in Newfoundland waters. ICES Journal of Marine Science, 66c:1800-1807. 